\title{
THE ABUNDANCE SPREAD IN THE BOÖTES I DWARF SPHEROIDAL GALAXY
}

\author{
John E. Norris, ${ }^{1}$ Gerard Gilmore, ${ }^{2}$ Rosemary F. G. Wyse, ${ }^{3}$ Mark I. Wilkinson, ${ }^{2,4}$ V. Belokurov, ${ }^{2}$ \\ N. Wyn Evans, ${ }^{2}$ AND DANIEl B. ZuCKeR ${ }^{2}$ \\ Received 2008 September 1; accepted 2008 October 29; published 2008 November 14
}

\begin{abstract}
We present medium-resolution spectra of 16 radial velocity red-giant members of the low-luminosity Boötes I dwarf spheroidal (dSph) galaxy that have sufficient $\mathrm{S} / \mathrm{N}$ for abundance determination, based on the strength of the $\mathrm{Ca}$ II $\mathrm{K}$ line. Assuming $[\mathrm{Ca} / \mathrm{Fe}] \sim 0.3$, the abundance range in the sample is $\Delta[\mathrm{Fe} / \mathrm{H}] \sim 1.7 \mathrm{dex}$, with one star having $[\mathrm{Fe} / \mathrm{H}]=-3.4$. The dispersion is $\sigma([\mathrm{Fe} / \mathrm{H}])=0.45 \pm 0.08$-similar to those of the Galaxy's more luminous dSph systems and $\omega$ Centauri. This suggests that the large mass $\left(\gtrsim 10^{7} M_{\odot}\right)$ normally assumed to foster self-enrichment and the production of chemical abundance spreads was provided by the nonbaryonic material in Boötes I.
\end{abstract}

Subject headings: galaxies: abundances — galaxies: dwarf — galaxies: individual (Boötes I)

Online material: color figures

\section{INTRODUCTION}

The Galaxy's dSph satellites continue to play a challenging and pivotal role in our understanding of the $\Lambda \mathrm{CDM}$ paradigm and the manner in which the Milky Way formed. Not only do there appear to be too few of these systems in comparison with the predictions of CDM satellite dark halos (Klypin et al. 1999; Moore et al. 1999), but for those who see the dSphs as possible building blocks for the Galaxy's halo (e.g., Carollo et al. 2007), their stellar populations pose problems. Not only are their age distributions too young (Unavane et al. 1996), their chemistry, at first blush, is wrong, at least as far as, e.g., $[\alpha / \mathrm{Fe}]$ is concerned (Venn et al. 2004 and references therein). The report by Helmi et al. (2006) of a dearth of stars with $[\mathrm{Fe} / \mathrm{H}]<-3.0$ in the more luminous dSphs, if correct, offers no comfort to those who seek the origin of the now well-established Galactic extremely metal-poor stars having $-4.0<[\mathrm{Fe} / \mathrm{H}]<-3.0$.

The recent discovery of very low luminosity dSph galaxies, down to $M_{V} \sim-4$ (e.g., Belokurov et al. 2006, 2007), but with associated nonbaryonic masses of $10^{7} M_{\odot}$ within the extent of the stellar distribution (e.g., Gilmore et al. 2007) hints there is still much to be understood before we have a complete understanding of the process of the formation of our Galaxy.

An important feature of the more luminous of the Milky Way's dSphs is that there exists a well-established range in metallicity within each system, with dispersions of order 0.30.5 dex (e.g., Helmi et al. 2006). ${ }^{5}$ The purpose of this Letter is to present evidence that similar behavior pertains to one of the less luminous systems, Boötes I, with $M_{V}=-5.8$ (Belokurov et al. 2006). We shall argue that Boötes I exhibits a range in metallicity of $\sim 1.7 \mathrm{dex}, \sigma([\mathrm{Fe} / \mathrm{H}])=0.45$, and stars as metalpoor as $[\mathrm{Fe} / \mathrm{H}]=-3.4$.

\footnotetext{
' Research School of Astronomy and Astrophysics, Australian National University, Mount Stromlo Observatory, Cotter Road, Weston, ACT 2611, Australia; jen@mso.anu.edu.au.

${ }^{2}$ Institute of Astronomy, University of Cambridge, Madingley Road, Cambridge CB3 0HA, UK.

${ }^{3}$ Johns Hopkins University, Department of Physics and Astronomy, 3900 North Charles Street, Baltimore, MD 21218.

${ }^{4}$ Department of Physics and Astronomy, University of Leicester, University Road, Leicester LE1 7RH, UK.

${ }^{5}$ Kirby et al. (2008) have very recently reported abundance spreads in several of the Galaxy's less luminous dSph systems.
}

\section{SPECTROGRAPHIC OBSERVATIONS AND DATA REDUCTION}

Spectra of $\sim 330$ Boötes I candidate members were obtained with the Anglo-Australian Telescope/ AAOmega fiber-fed spectrograph combination ${ }^{6}$ during 2006 May 23-29 and 2007 April $18-20$. In the blue, the spectra have resolution $R=5000$, and cover the wavelength range $3850-4540 \AA$, while in the red the corresponding numbers are 14000 and 8340-8840 $\AA$, respectively.

Candidates were selected from the SDSS DR4 data set, using the color-magnitude diagram selection mask illustrated in Figure 2 of Belokurov et al. (2006). Stars in the magnitude range $17<g<20.5$ were selected for observation with AAOmega, with a deliberate effort to select candidates up to $1^{\circ}$ from the galaxy center, four half-light radii, given the galaxy's apparently distorted and elliptical image. Only limited observational material was obtained in 2006. Deeper data, using essentially the same target selection, were obtained in 2007. The 2006 run was the first major visitor use of the new AAOmega facility. These data sets were used to optimize and enhance the data reduction system, $2 \mathrm{dfdr}{ }^{7}$ in collaboration with local expertise, leading to its current excellent performance. Final data calibration and reduction used what is now the public $2 \mathrm{dfdr}$ system.

\section{ANALYSIS}

\subsection{Radial Velocities}

Heliocentric radial velocities were determined using the HCROSS routine of the FIGARO package. ${ }^{8}$ This performs a cross-correlation between the program stellar spectrum and a template, to obtain the relative radial velocity. An associated confidence level and formal error are estimated and we accept only those with confidence $=1$ (see Heavens 1993). We excised the strong $\mathrm{Ca}$ II $\mathrm{H}$ and $\mathrm{K}$ lines, although this made an insignificant difference in most cases. Two template spectra were used, of twilight sky and a G-giant star, both obtained during the same observing run as the Boötes I candidates. The velocities from the two templates were in general consistent within the errors and here we report those using twilight sky as template. We thus obtained velocities for 98 objects, for which our mean formal error is $3.6 \mathrm{~km} \mathrm{~s}^{-1}$. The internal ac-

\footnotetext{
${ }^{6}$ See http://www.aao.gov.au/local/www/aaomega/.

${ }^{7}$ See http://www.aao.gov.au/AAO/2df/software.html\#2dfdr.

${ }^{8}$ See http://www.starlink.rl.ac.uk/star/docs/sun86.htx/node425.html.
} 
TABLE 1

Observational Data and Derived Parameters for Boötes I

\begin{tabular}{|c|c|c|c|c|c|c|c|c|c|c|c|}
\hline $\begin{array}{l}\text { Star } \\
(1)\end{array}$ & $\begin{array}{c}\text { R.A. } \\
\text { (J2000.0) } \\
(2)\end{array}$ & $\begin{array}{c}\text { Decl. } \\
\text { (J2000.0) } \\
(3)\end{array}$ & $\begin{array}{c}r \\
(\operatorname{arcmin}) \\
(4)\end{array}$ & $\begin{array}{c}V_{r} \\
\left(\mathrm{~km} \mathrm{~s}^{-1}\right) \\
(5)\end{array}$ & $\begin{array}{l}g_{0} \\
(6)\end{array}$ & $\begin{array}{c}(g-r)_{0} \\
(7)\end{array}$ & $\begin{array}{c}\text { C4150 } \\
(8)\end{array}$ & $\begin{array}{l}K^{\prime} \\
(\AA) \\
(9)\end{array}$ & $\begin{array}{c}G^{\prime} \\
(\AA) \\
(10)\end{array}$ & $\begin{array}{c}{[\mathrm{Fe} / \mathrm{H}]} \\
(11)\end{array}$ & $\begin{array}{c}\text { W8552 } \\
(\AA) \\
(12)\end{array}$ \\
\hline & 135935.53 & +142023.7 & 12.1 & 104 & 18.31 & 0.70 & 609 & 7.49 & 2.01 & -2.32 & 1.35 \\
\hline $8 \ldots \ldots \ldots$ & 135938.62 & +141915.9 & 12.6 & 109 & 19.03 & 0.56 & 345 & 4.53 & 3.25 & -2.75 & 0.84 \\
\hline $9 \ldots \ldots \ldots$ & 135948.81 & +141942.9 & 11.1 & 108 & 17.92 & 0.80 & 772 & 6.62 & 1.99 & -2.67 & 1.16 \\
\hline $33 .$. & $1400 \quad 11.73$ & +142501.4 & 5.2 & 105 & 18.23 & 0.74 & 321 & 5.17 & 5.28 & -2.96 & 1.20 \\
\hline $34 \ldots$ & 140021.11 & +141727.9 & 13.1 & 104 & 18.74 & 0.65 & 313 & 4.94 & 2.66 & -3.10 & 1.20 \\
\hline $41 \ldots$ & $1400 \quad 25.83$ & +142607.6 & 6.2 & 98 & 18.38 & 0.70 & 239 & 8.13 & 4.29 & -2.03 & 2.17 \\
\hline \multirow[t]{2}{*}{$78 \ldots \ldots \ldots$} & 140014.73 & +141313.9 & 16.9 & 102 & 19.30 & 0.64 & 237 & 6.10 & 2.24 & -2.46 & 1.14 \\
\hline & & & & & & & 202 & 4.88 & 1.48 & -3.02 & 1.25 \\
\hline $94 \ldots$ & 140031.51 & +143403.6 & 7.4 & 93 & 17.53 & 0.90 & 538 & 6.63 & 0.14 & -2.79 & 1.24 \\
\hline 117 & $14 \quad 00 \quad 10.49$ & +143145.5 & 2.1 & 94 & 18.19 & 0.74 & 283 & 8.78 & 3.46 & -1.72 & 1.46 \\
\hline \multirow[t]{2}{*}{$121 \ldots \ldots$} & 140036.52 & +143927.3 & 12.0 & 113 & 17.92 & 0.83 & 237 & 7.62 & 3.23 & -2.37 & 1.51 \\
\hline & & & & & & & 387 & 7.43 & 3.14 & -2.46 & 1.54 \\
\hline \multirow[t]{2}{*}{$127 \ldots \ldots$} & $\begin{array}{llll}14 & 00 & 14.57\end{array}$ & +143552.7 & 6.2 & 99 & 18.15 & 0.76 & 236 & 9.47 & 4.72 & -1.49 & 1.67 \\
\hline & & & & & & & 346 & 9.36 & 3.96 & -1.49 & 1.86 \\
\hline 130 & 135948.98 & +143006.2 & 4.1 & 110 & 18.21 & 0.70 & 337 & 6.60 & 2.13 & -2.55 & 1.22 \\
\hline 911 & 140001.08 & +143651.5 & 7.0 & 97 & 17.94 & 0.75 & 237 & 8.47 & 3.44 & -1.98 & 1.51 \\
\hline 980 & 135912.68 & +134255.8 & 48.8 & 104 & 18.51 & 0.61 & 396 & 4.14 & 0.82 & -3.09 & 0.86 \\
\hline \multirow[t]{2}{*}{1069.} & 135853.22 & +140657.8 & 29.0 & 111 & 19.05 & 0.56 & 490 & 5.57 & 1.27 & -2.51 & 0.96 \\
\hline & & & & & & & 200 & 3.80 & 1.52 & -2.91 & \\
\hline $1137 \ldots \ldots$ & 135833.82 & +142108.5 & 24.0 & 112 & 18.11 & 0.73 & 859 & 3.06 & 1.09 & -3.45 & 0.66 \\
\hline
\end{tabular}

curacy on one observation, from repeat observations of four stars from 2006 and 2007, is $7 \mathrm{~km} \mathrm{~s}^{-1}$ with a mean offset of $-1.5 \mathrm{~km} \mathrm{~s}^{-1}$. Our external errors may be estimated by comparison with Martin et al. (2007). For five stars in common, observed by us in 2007, the mean offset is $4.6 \mathrm{~km} \mathrm{~s}^{-1}$, with $\sigma$ $=1.8 \mathrm{~km} \mathrm{~s}^{-1}$.

\subsection{Selection of Boötes I Members}

For the purposes of the present work we choose to consider objects for abundance analysis only if their spectra have net counts greater than $\sim 200$ per $0.34 \AA$ pixel at $4150 \AA$ and lie within the radial velocity range $90-115 \mathrm{~km} \mathrm{~s}^{-1}$. The first criterion is set to obtain a reliable abundance estimate based on the Ca II K $3933 \AA$ line, while the second admits objects within $\pm 1.8 \sigma$ of the galaxy's systemic velocity (we adopt a velocity dispersion of $7 \mathrm{~km} \mathrm{~s}^{-1}$ ). Data for the 16 objects within our sample that satisfy these criteria are presented in Table 1, where columns (1)-(5) contain identification, coordinates, radial distance from galactic center, and heliocentric radial velocity, (6)(7) present SDSS colors $g_{0}$ and $(g-r)_{0}$ [we adopt $E(B-$ $V)=0.02$, following Belokurov et al. 2006], and column (8) contains the net counts obtained per $0.34 \AA$ pixel at $4150 \AA$. For four objects, data are available from both the 2006 and 2007 sessions - in these cases the second entry in the table refers to data obtained in 2006.

We believe our sample has very small field contamination. In velocity windows at lower (65-90 $\left.\mathrm{km} \mathrm{s}^{-1}\right)$ and higher (115$140 \mathrm{~km} \mathrm{~s}^{-1}$ ) values, and for our count restriction, we find a total of four objects (one clearly too strong-lined to be a member). Standard Galactic components of thin disk, thick disk, and halo have predicted mean velocities in this line of sight, for a typical distance of dwarf stars of $3 \mathrm{kpc}$, of $-5,-25$, and $-130 \mathrm{~km} \mathrm{~s}^{-1}$, respectively, well separated from Boötes I.

\subsection{Chemical Abundances from the Ca II $K$ line}

For the putative Boötes I members in Table 1 we have measured the Ca II K line-strength index, $K^{\prime}$, and the $G$-band index, $G^{\prime}$, defined by Beers et al. (1999), which we present in columns (9) and (10) of Table 1. Beers et al. provide a formalism, based on Galactic field stars and globular clusters that permits one to estimate $[\mathrm{Fe} / \mathrm{H}]$ given $K^{\prime}$ and $(B-V)_{0}$, well calibrated down to $[\mathrm{Fe} / \mathrm{H}]=-4.0$, which we adopt here. The reader should be aware that the implicit assumption, that the same $[\mathrm{Ca} / \mathrm{Fe}]$ versus $[\mathrm{Fe} / \mathrm{H}]$ relationship applies within both the metal-poor Galactic objects used for the calibration and the dSph satellites, is not consistent with high-resolution abundance studies (e.g., Venn et al. 2004) at, say, the 0.3 dex level for the dSph systems studied to date. Here we shall cite $[\mathrm{Fe} / \mathrm{H}]$ values obtained from the Beers et al. calibration, assuming $[\mathrm{Ca} / \mathrm{Fe}] \sim 0.3$; allowance for a putative spread in $[\mathrm{Ca} / \mathrm{Fe}]$ in Boötes I would increase any inferred dispersion of $[\mathrm{Fe} / \mathrm{H}]$. To use the Beers et al. formulation we have obtained $B-V$ values using the transformation $(B-V)_{0}=1.197 \times(g-r)_{0}+0.049$ valid on the range $0.70<B-V<1.3$, determined from observations of the red giants in the Galactic globular clusters M13 and NGC 2419.

The resulting $[\mathrm{Fe} / \mathrm{H}]$ values are presented in column (11) of Table 1. We find $\langle[\mathrm{Fe} / \mathrm{H}]\rangle=-2.51 \pm 0.13$, which agrees well with Muñoz et al. (2006, $[\mathrm{Fe} / \mathrm{H}]=-2.5)$, Martin et al. (2007, $[\mathrm{Fe} / \mathrm{H}]=-2.1)$, and S. Feltzing et al. $\left(2008,{ }^{10}[\mathrm{Fe} / \mathrm{H}]=-2.7\right)$. Small-sample statistics (Keeping 1962) of the abundance differences for the four stars in Table 1 observed in both 2006 and 2007 yields a standard deviation of a single observation of 0.19 dex, ${ }^{11}$ while comparison of results for seven stars in common with S. Feltzing et al. (assuming equal errors in their work and ours) yields 0.35 dex. Further consideration of Table 1 reveals the principal result of our investigation: among the 16 putative galaxy members there is a spread of $\Delta[\mathrm{Fe} / \mathrm{H}] \sim$

\footnotetext{
${ }^{9}$ Based on data kindly made available to us by H. L. Morrison $(B-V$ and $g-r$ photometry of P. Stetson [2008, http://www1.cadc-ccda.hia-iha.nrccnrc.gc.ca/community/STETSON/standards/] and An et al. [2008], and cluster membership for NGC 2419 from Peterson [1985], Shetrone et al. [2001], and Suntzeff et al. [1988]). We prefer this calibration to those of R. Lupton (2005, http://www.sdss.org/dr5/algorithms/sdssUBVRITransform.html) and Zhao \& Newberg (2006), from stars in the general field and metal-poor objects having $B-V<0.70$, respectively.

${ }^{10}$ See http://www.mpa-garching.mpg.de/ garcon08/program.html.

${ }^{11}$ A second estimate of our internal accuracy comes from $\sim 10,000$ dwarfs observed during our 2006 session (and analyzed with similar techniques), which serendipitously included 16 objects with $[\mathrm{Fe} / \mathrm{H}]<-1.5$, and $g>18.0$, from SSDS DR6 (see Lee et al. 2008). The dispersion of abundance differences between the two subsamples (over 8 independent pointings) is $0.23 \mathrm{dex}$.
} 

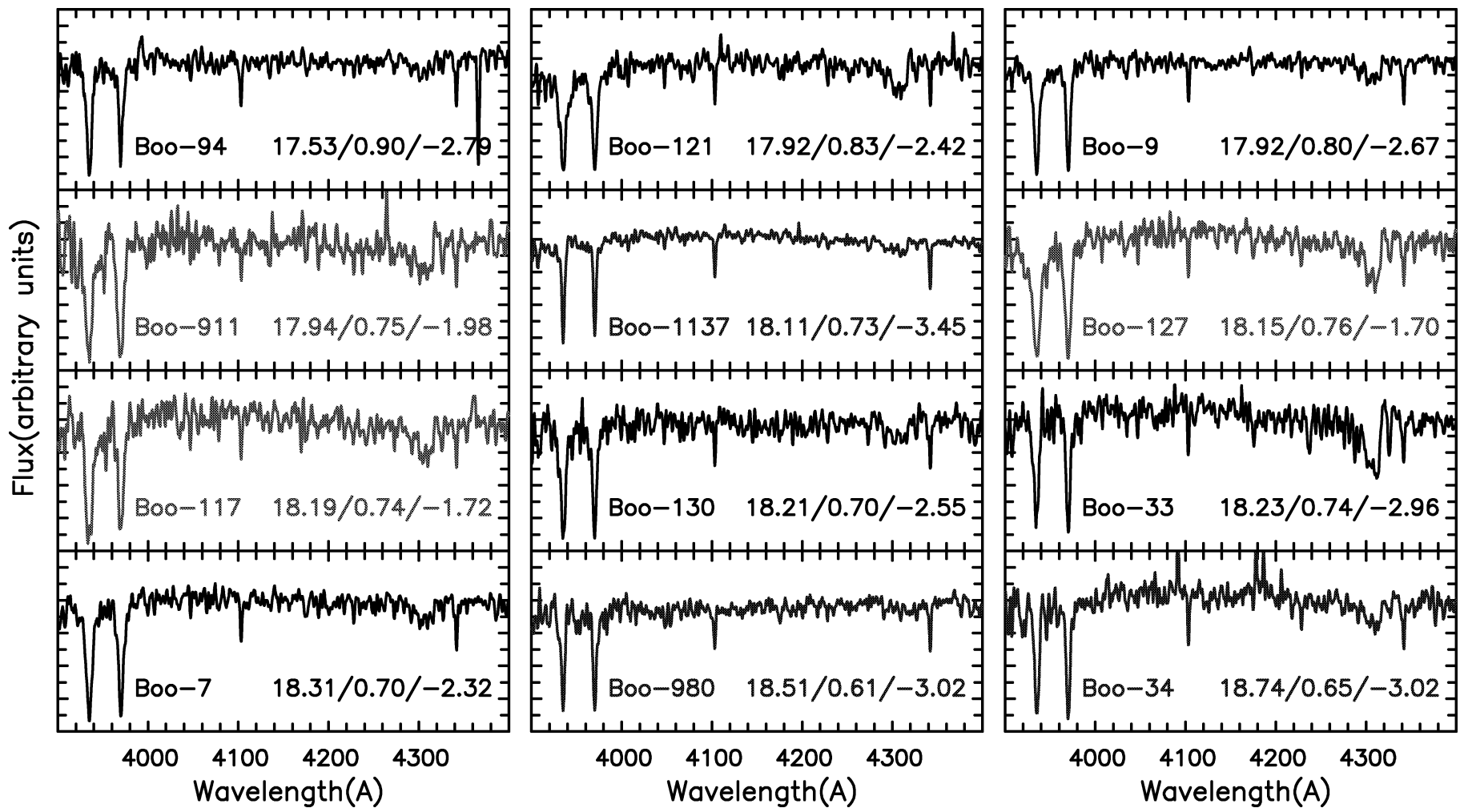

FIG. 1.-Spectra of 15 Boötes I red giants from Table 1 (continuum-normalized and broadened to resolution of FWHM $=2.5 \AA$ ). Panels contain star name

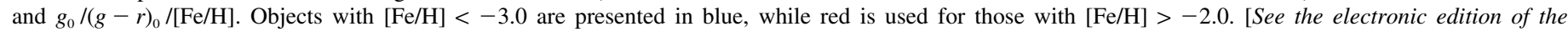
Journal for a color version of this figure.]

1.7-considerably larger than expected from the above measurement accuracies. The abundance dispersion for our 2007 data, corrected for measurement error of $0.19(0.35)$ dex is 0.49 (0.40): we adopt $\sigma[\mathrm{Fe} / \mathrm{H}]=0.45 \pm 0.08$ for Boötes $\mathrm{I}$.

The large range in abundance can also be appreciated in Figure 1, which presents the spectra for 15 stars in Table 1. Figure 2 compares the most metal-poor object in our sample (Boo 1137, with $[\mathrm{Fe} / \mathrm{H}]=-3.4$ ) with classical metal-poor stars in the range $-4.2<[\mathrm{Fe} / \mathrm{H}]<-2.5$. The low metallicity of Boo 1137 is well-confirmed. ${ }^{12}$

\subsection{The Calcium Triplet}

To complement the $\mathrm{Ca}$ II $\mathrm{K}$ line data we present equivalent widths of the Ca II triplet $8542 \AA$ line in column (12) of Table 1. (Poor sky subtraction prevents measurement of the triplet 8662 $\AA$ line.) There is a clear and strong correlation between the $\mathrm{K}$ line and triplet line data in Table 1 . The question that needs to be addressed is whether the triplet data support the $\mathrm{K}$ line abundances.

To our knowledge, all abundance calibrations based on the triplet follow Armandroff \& Da Costa (1991, hereafter AD91) and are anchored to the abundances of Galactic globular clusters, none of which has $[\mathrm{Fe} / \mathrm{H}]<-2.5$. (This method also assumes $[\mathrm{Ca} / \mathrm{Fe}] \sim 0.3$.) Extrapolation is thus necessary when using the triplet for the $\sim$ half of the objects in Table 1 having $[\mathrm{Fe} / \mathrm{H}]<$

\footnotetext{
${ }^{12}$ Boo 1137 lies $24^{\prime}$ from the center of the system, well outside the halflight radius of 13' (but along the major axis of the galaxy's elongation). Given the rareness of objects with $[\mathrm{Fe} / \mathrm{H}]<-3.0$, however, it is very likely to be a member. From the HK survey for metal-poor stars, Beers et al. (1992) find 5 stars with $[\mathrm{Fe} / \mathrm{H}]<-3.0, V<14$, and $B-V>0.7$, over $2300 \mathrm{deg}^{2}$. For the HES, N. Christlieb (2008, private communication) finds 9 stars with $B<$ 14.5 and $B-V>0.7$, over $7000 \mathrm{deg}^{2}$. We estimate one should expect only $\sim 0.02$ halo giants having $[\mathrm{Fe} / \mathrm{H}]<-3.0$ within the central $30^{\prime}$ of Boötes I.
}

-2.5 . To make progress we proceed as follows. Based on spectra of standard globular cluster giants with $[\mathrm{Fe} / \mathrm{H}]<-1.4$ described by Norris et al. (1996), we transform $W_{8542}$ to the AD91 triplet index $W_{\mathrm{AD}}=W_{8542}+W_{8662}$ using the relationship $W_{\mathrm{AD}}=$ $2.34 \times W_{8542}$. The pivotal diagram of AD91 is their plot of $W_{\mathrm{AD}}$ versus $V-V_{\mathrm{HB}}\left(V\right.$ is the star's magnitude and $V_{\mathrm{HB}}$ refers to the horizontal branch of the cluster under consideration). To obtain $V$ we transform the $g_{0}$ and $(g-r)_{0}$ values in Table 1 following R. Lupton (2005; see footnote 9 above). For Boötes I, $V_{\mathrm{HB}}=$ 19.55 (Dall'Ora et al. 2006). All but two of the objects in Table 1 fall below the M15 $([\mathrm{Fe} / \mathrm{H}]=-2.2)$ data of AD91 (their Fig. 3 ) in their uncalibrated region, reasonably consistent with only four stars in our Table 1 having $[\mathrm{Fe} / \mathrm{H}]>-2.2$.

AD91 also define the reduced index $W^{\prime}=W_{8542}+W_{8662}+$ $0.619 \times\left(V-V_{\mathrm{HB}}\right)$, and determine the calibration $[\mathrm{Fe} / \mathrm{H}]=[\mathrm{Fe} /$ $\mathrm{H}]_{\mathrm{AD}} \equiv 0.326 \times W^{\prime}-2.706$, valid for $1.6<W^{\prime}<4.2(-2.2<$ $[\mathrm{Fe} / \mathrm{H}]<-1.3)$. Using these relationships for the data in Table 1, we obtain $\langle[\mathrm{Fe} / \mathrm{H}]\rangle_{\mathrm{AD}}=-2.32$ and $\sigma[\mathrm{Fe} / \mathrm{H}]_{\mathrm{AD}}=0.20$, somewhat different from the values obtained from the Ca II $\mathrm{K}$ line. For Boo 1137, with $[\mathrm{Fe} / \mathrm{H}]_{\text {CaII K }}=-3.4$, we find $[\mathrm{Fe} / \mathrm{H}]_{\text {Ca I t triplet }}=-2.7$.

We found no model-atmosphere calibrations of the triplet in the literature for giants with $[\mathrm{Fe} / \mathrm{H}]<-2.5$. This is perhaps not surprising: in this regime the triplet is relatively weak, core-dominated, and challenging to model atmosphere analysis. Using the spectrum synthesis code MOOG of C. Sneden (2008), models of Kurucz (1993), atomic data from VALD, and a range of van der Waals damping values for the triplet, together with $T_{\text {eff }}, \log g$, and colors from the Yale-Yonsei isochrones ${ }^{13}$ we computed spectra for $[\mathrm{Fe} / \mathrm{H}]=-1.5$ to -4.0 , and plotted isoabundant loci in the

\footnotetext{
${ }^{13}$ See http://verdi.as.utexas.edu/moog.html, http://www.astro.uu.se/ vald/, and http://www.astro.yale.edu/demarque/yyiso.html, respectively.
} 


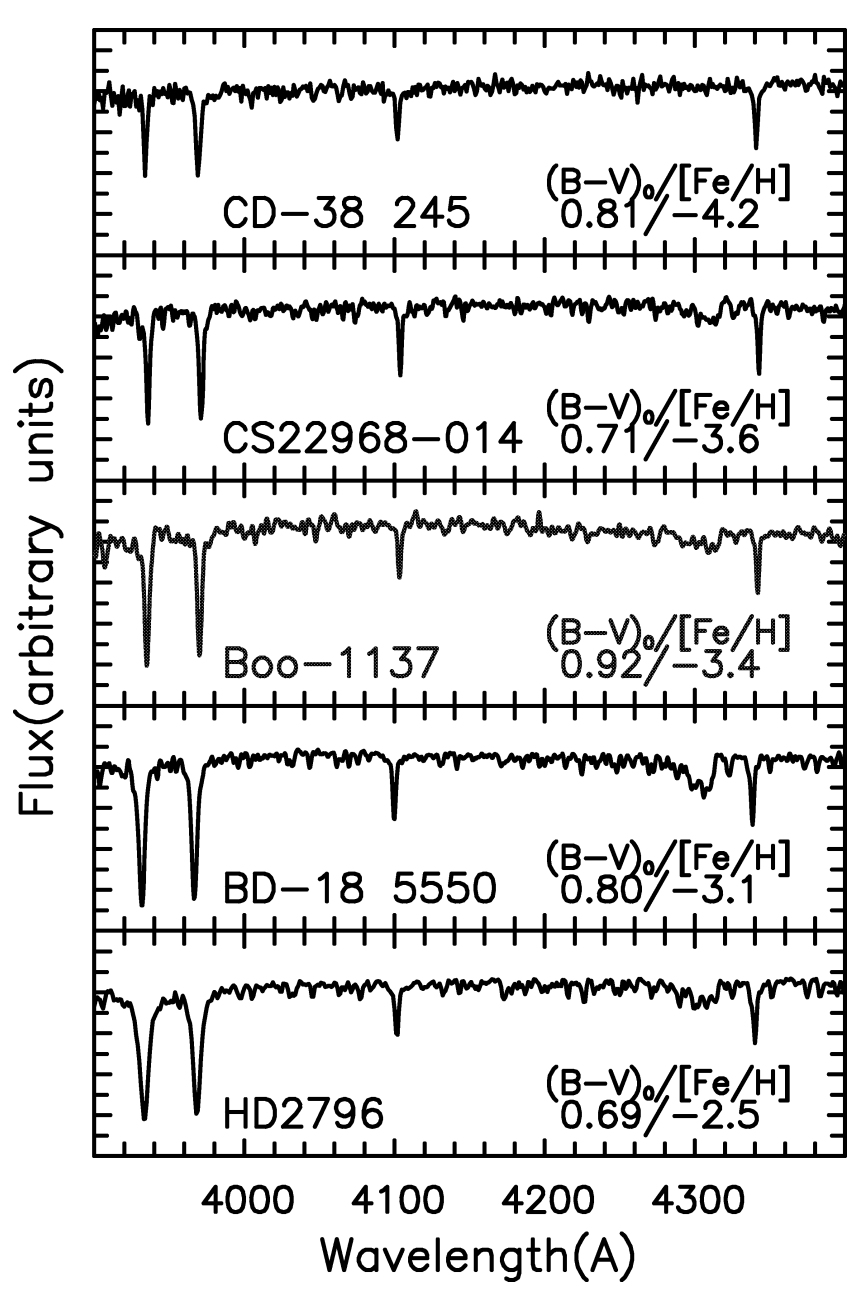

FIG. 2.-Spectra of Boo $1137([\mathrm{Fe} / \mathrm{H}]=-3.4$; blue line $)$ and extremely metal-poor field giants of similar temperature (from ANU's $2.3 \mathrm{~m}$ telescope; $[\mathrm{Fe} / \mathrm{H}]$ and $(B-V)_{0}$ from Cayrel et al. [2004] and Beers et al. [1999], respectively). All comparison stars are hotter than Boo 1137, and would have slightly stronger Ca II K $3933 \AA$ lines than seen here, if they were as cool as it. [See the electronic edition of the Journal for a color version of this figure.]

$W_{\mathrm{AD}}$ versus $\left(V-V_{\mathrm{HB}}\right)$ plane. The agreement between observation and theory leaves something to be desired: while the observed loci are essentially parallel, as are the theoretical ones, the latter have a shallower slope. Most importantly, the theoretical lines move closer together at lowest abundance. We use these results to indicate, approximately, the improvement we believe necessary to extrapolate the AD91 relationship. For $[\mathrm{Fe} / \mathrm{H}]>-2.5$, we adopt $[\mathrm{Fe} / \mathrm{H}]=[\mathrm{Fe} / \mathrm{H}]_{\mathrm{AD}}=0.326 \times W^{\prime}-2.706$, and, for $-4.0<[\mathrm{Fe} /$ $\mathrm{H}]<-2.5,[\mathrm{Fe} / \mathrm{H}]=[\mathrm{Fe} / \mathrm{H}]_{\mathrm{AD}}-1.03+0.83 \times W^{\prime}-$ $0.15 \times W^{\prime 2}$. Then for the data in Table 1 , we find $\langle[\mathrm{Fe} / \mathrm{H}]\rangle=$ $-2.64, \sigma[\mathrm{Fe} / \mathrm{H}]=0.45$, and $[\mathrm{Fe} / \mathrm{H}]_{\mathrm{Boo} 1137}=-3.8$.

\section{DISCUSSION}

Despite its small baryonic mass, Boötes I has an abundance dispersion very comparable with those of the more massive dSphs $(\sigma[\mathrm{Fe} / \mathrm{H}]=0.3-0.6$; e.g., Helmi et al. 2006) and the most massive $\left(3 \times 10^{6} M_{\odot}\right)$ Galactic globular cluster $\omega$ Cen $(\sigma[\mathrm{Fe} / \mathrm{H}]=0.3$; Norris et al. 1996). With a current stellar mass of $\sim 4 \times 10^{4}$ $M_{\odot}$ (Fellhauer et al. 2008), however, the chemical inhomogeneity of Boötes I stands in stark contrast against the heavy-element homogeneity of Galactic globular clusters of similar stellar mass. The obvious solution is that Boötes I sits within the deep potential well of a dark halo mass — of order $10^{7}-10^{8} M_{\odot}$. Given that chemical inhomogeneity within globular clusters (which contain no dark mass) appears to occur only in massive objects such as $\omega$ Cen, it seems reasonable to assume that a requirement for the creation of heavy-element chemical inhomogeneity is that Boötes I should have a potential well deep enough to retain the ejecta of Type II supernovae for periods sufficiently long for them to be incorporated into subsequent stellar generations.

There is a test of this: self-enrichment in the lowest luminosity systems will be greatly affected by sampling noise. As demonstrated by the dynamical modeling of Fellhauer et al. (2008), if Boötes I ever had a dark matter halo, it still has it and has lost very little of its stellar mass. That is to say, Boötes I has always had relatively little stellar material. If one normalizes abundance dispersion with respect to luminosity or current baryonic mass, the specific abundance dispersion of Boötes I is some 10-100 times larger than that of the more luminous dSph. Not many supernovae are needed to chemically enrich, say, $10^{5} M_{\odot}$ of pristine material to an abundance of $[\mathrm{Fe} / \mathrm{H}]=-2.5$. One should expect very specific and different element patterns in the stars of Boötes I directly from this very small number of progenitors. One may be looking at the yields of individual supernovae in the abundances of these stars.

The authors gratefully acknowledge the contributions of the AAOmega project team, in particular Rob Sharp, during this investigation. We thank $\mathrm{H}$. L. Morrison for generous instruction in $(B-V, g-r)$-transformations.

\section{REFERENCES}

An, D., et al. 2008, arXiv:0808.0001 (SDSS paper 869)

Armandroff, T. E., \& Da Costa, G. S. 1991, AJ, 101, 1329 (AD91)

Beers, T. C., Preston, G. W., \& Shectman, S. A. 1992, AJ, 103, 1987

Beers, T. C., et al. 1999, AJ, 117, 981

Belokurov, V., et al. 2006, ApJ, 647, L111 2007, ApJ, 654, 897

Carollo, D., et al. 2007, Nature, 450, 1020

Cayrel, R., et al. 2004, A\&A, 416, 1117

Dall'Ora, M., et al. 2006, ApJ, 653, L109

Fellhauer, M., et al. 2008, MNRAS, 385, 1095

Gilmore, G., et al. 2007, ApJ, 663, 948

Heavens, A. 1993, MNRAS, 263, 735

Helmi, A., et al. 2006, ApJ, 651, L121

Keeping, E. S. 1962, Introduction to Statistical Inference (Princeton: Van Nostrand), 202
Kirby, E. N., et al. 2008, ApJ, 685, L43

Klypin, A., et al. 1999, ApJ, 522, 82

Kurucz, R. L. 1993, CD-ROM 13, ATLAS9 Stellar Atmospheres Programs and $2 \mathrm{~km} / \mathrm{s}$ Grid (Cambridge: SAO)

Lee, Y. S., et al. 2008, AJ, 136, 2022

Martin, N. F., et al. 2007, MNRAS, 380, 281

Moore, B., et al. 1999, ApJ, 524, L19

Muñoz, R. R., et al. 2006, ApJ, 650, L51

Norris, J. E., Freeman, K. C., \& Mighell, K. J. 1996, ApJ, 462, 241

Peterson, R. C. 1985, ApJ, 297, 309

Shetrone, M. D., Côte, P., \& Sargent, W. L. W. 2001, ApJ, 548, 592

Suntzeff, N. B., Kraft, R. P., \& Kinman, T. D. 1988, AJ, 95, 91

Unavane, M., Wyse, R. F. G., \& Gilmore, G. 1996, MNRAS, 278, 727

Venn, K. A., et al. 2004, AJ, 128, 1177

Zhao, C., \& Newberg, H. J. 2006, preprint (astro-ph/0612034) 